\title{
Lineage-specific exosomes promote the odontogenic differentiation of human dental pulp stem cells (DPSCs) through TGF $\beta 1 /$ smads signaling pathway via transfer of microRNAs
}

Xiaoli Hu ${ }^{1,2^{*}+}$, Yingqun Zhong ${ }^{1,2+}$, Yuanyuan Kong ${ }^{3,4}$, Yanan Chen ${ }^{1,2}$, Junming Feng ${ }^{1,2}$ and Jianmao Zheng ${ }^{1,2^{*}}$ (D)

\begin{abstract}
Background: Exosomes derived from dental pulp stem cells (DPSCs) can be used as biomimetic tools to induce odontogenic differentiation of stem cells, but the regulatory mechanisms and functions of exosome-encapsulated microRNAs are still unknown. The present study aimed to clarify the role of microRNAs contained in the exosomes derived from human DPSCs and their potential signaling cascade in odontogenic differentiation.

Methods: Exosomes were isolated from human DPSCs cultured undergrowth and odontogenic differentiation conditions, named UN-EXO and OD-ExO, respectively. The microRNA sequencing was performed to explore the microRNA profile contained in UN-Exo and OD-Exo. Pathway analysis was taken to detect enriched pathways associated with the predicted target genes of microRNAs. The regulatory roles of a highly expressed microRNA in OD-Exo were investigated through its inhibition or overexpression (miRNA inhibitors and miRNA mimics). Automated western blot was used to identify the function of exosomal microRNA and the roles of TGF $\beta 1 / \mathrm{smads}$ pathway in odontogenic differentiation of DPSCs. A luciferase reporter gene assay was used to verify the direct target gene of exosomal miR-27a-5p.
\end{abstract}

Results: Endocytosis of OD-Exo triggered odontogenic differentiation of DPSCs by upregulating DSP, DMP-1, ALP, and RUNX2 proteins. MicroRNA sequencing showed that 28 microRNAs significantly changed in OD-Exo, of which 7 increased and 21 decreased. Pathway analysis showed genes targeted by differentially expressed microRNAs were involved in multiple signal transductions, including TGF $\beta$ pathway. 16 genes targeted by 15 differentially expressed microRNAs were involved in TGF $\beta$ signaling. Consistently, automated western blot found that OD-Exo activated TGF $\beta 1$ pathway by upregulating TGF $\beta 1$, TGFR1, p-Smad2/3, and Smad4 in DPSCs. Accordingly, once the TGF $\beta 1$ signaling pathway was inhibited by SB525334, protein levels of p-Smad2/3, DSP, and DMP-1 were significantly decreased in DPSCs treated with OD-Exo. MiR-27a-5p was expressed 11 times higher in OD-Exo, while miR-27a-5p promoted odontogenic differentiation of DPSCs and significantly upregulated TGF $\beta 1$, TGFR1, p-Smad2/3, and Smad4 by downregulating the inhibitory molecule LTBP1.

(Continued on next page)

\footnotetext{
*Correspondence: huxiaol3@mail.sysu.edu.cn; zhengjm25@mail.sysu.edu.cn

${ }^{+}$Xiaoli Hu and Yingqun Zhong contributed equally to this work.

${ }^{1}$ Department of Operative Dentistry and Endodontics, Guanghua School of

Stomatology, Affiliated Stomatological Hospital, Sun Yat-sen University,

Guangzhou 510055, Guangdong, China

Full list of author information is available at the end of the article
}

(c) The Author(s). 2019 Open Access This article is distributed under the terms of the Creative Commons Attribution 4.0 International License (http://creativecommons.org/licenses/by/4.0/), which permits unrestricted use, distribution, and reproduction in any medium, provided you give appropriate credit to the original author(s) and the source, provide a link to the Creative Commons license, and indicate if changes were made. The Creative Commons Public Domain Dedication waiver (http://creativecommons.org/publicdomain/zero/1.0/) applies to the data made available in this article, unless otherwise stated. 
(Continued from previous page)

Conclusions: The microRNA expression profiles of exosomes derived from DPSCs were identified. OD-Exo isolated under odontogenic conditions were better inducers of DPSC differentiation. Exosomal microRNAs promoted odontogenic differentiation via TGFß1/smads signaling pathway by downregulating LTBP1.

Keywords: Exosomes, TGF $\beta$ pathway, MicroRNAs, Odontogenesis, DPSCs

\section{Introduction}

Clinically relevant dental pulp tissue regeneration can serve as a treatment to replace existing root canal therapy used to treat necrotic permanent teeth [1]. Regenerative endodontic treatment is attempted by using a variety of mesenchymal stem cells (MSCs), growth factors, and biomaterials [2, 3]. Exosomes are reported as an ideal biomaterial in regenerative endodontic treatment, with the properties of immunomodulation [4, 5], promoting angiogenesis [6] and inducing differentiation of stem cells $[7,8]$.

Exosomes are nano-sized vesicles ranging from 30 to $150 \mathrm{~nm}$ in diameter, which are secreted by many cell types to mediate intercellular communication [9]. The cargo of exosomes is shown to contain both ubiquitous and cell type-specific biological molecules such as protein, mRNA, and microRNA [9]. It has been revealed that exosomal microRNAs are transferred between cells and can regulate the post-transcriptional gene expression in recipient cells [10]. microRNAs can negatively regulate gene expression at the post-transcriptional level by binding to their target mRNAs through base pairing to the 3 '-untranslated region (UTR), causing translational repression of the mRNA [11]. Exosomal microRNAs have been shown to be critical components in stem cell differentiation. For example, exosomal miR-320c enhanced chondrogenic differentiation of bone marrow MSCs by upregulating SOX9 and downregulating MMP13 expression [12]. Similarly, exosomal miRlet-7 has been shown to initiate the osteogenic differentiation of MSCs [13].

Exosomes derived from dental pulp stem cells (DPSCs) can be used as biomimetic tools to induce odontogenic differentiation of stem cells during dental pulp regeneration [14]; however, the function and regulatory mechanism of microRNAs encapsulated in exosomes derived from DPSCs is still unknown. To date, no study has been conducted on the microRNA expression profiles of exosomes derived from human DPSCs. The present study aimed to clarify the role of microRNAs, encapsulated in the exosomes from human DPSCs, and their potential signaling cascade in odontogenic differentiation.

\section{Materials and methods}

\section{Isolation and culture of human DPSCs}

All experimental protocols were approved by the Ethics Committee of Sun Yat-sen University. Human DPSCs were harvested from healthy pulp tissues isolated from caries-free teeth of patients ( 5 females, age 24 35 years; 5 males, age 22 36 years) undergoing extraction of fully erupted third molars. Healthy pulp tissues were digested for isolation of DPSCs as described previously [15]. Cells were cultured at $37{ }^{\circ} \mathrm{C}$, in a $5 \% \mathrm{CO}_{2}$ incubator, using aMEM supplemented with 10\% FBS (GIBCO, USA) as a growth medium, $10 \mathrm{mg} / \mathrm{ml}$ streptomycin, and $10 \mathrm{U} / \mathrm{ml}$ of penicillin (Sigma, USA). Experiments were performed with DPSCs from passages 3 to 7.

\section{Investigation of DPSC surface markers}

$100 \mu \mathrm{l}$ DPSCs at a concentration of $1 \times 10^{6} \mathrm{cells} / \mathrm{ml}$ were stained by $5 \mu \mathrm{l}$ of each of the following human antibodies: CD45-PE, CD73-PE, CD90-APC, and CD166-PE (BD, USA). The samples were incubated at $37{ }^{\circ} \mathrm{C}$ for 30 min, centrifuged, washed twice with PBS, and examined by flow cytometry (BD, USA).

\section{Determination of DPSC differentiation capacity}

We determined the multi-potential differentiation of DPSCs into osteoblasts, adipocytes, and chondrocytes in vitro. To explore the potential of differentiation into osteoblasts, DPSCs were induced for 14 days in osteogenic medium supplemented with $100 \mathrm{nmol}$ dexamethasone, $10 \mathrm{mmol} \beta$-glycerophosphate, and $0.2 \mathrm{mmol} \mathrm{L}$-ascorbic acid (Sigma, USA), osteogenic differentiation was measured by Alizarin Red S staining. To verify the adipogenic differentiation potential, DPSCs were induced for 27 days in adipogenic medium supplemented with $0.5 \mu \mathrm{M}$ isobutyl-methylxanthine, $50 \mu \mathrm{M}$ indomethacin, $0.5 \mu \mathrm{M}$ dexamethasone, and $5 \mu \mathrm{g} / \mathrm{mL}$ insulin (Sigma, USA), adipogenic differentiation was determined by Oil Red O staining. For chondrogenic differentiation, the cell pellets were prepared for a three-dimensional culture system. Approximately $4 \times 10^{5}$ cells were placed in a 15 $\mathrm{ml}$ polypropylene tube and centrifuged at $500 \mathrm{~g}$ for $5 \mathrm{~min}$. The cells were cultured in human mesenchymal stem cell chondrogenic differentiation medium (Cyagen, USA) for 28 days, in the presence of $10 \mathrm{ng} / \mathrm{mL}$ recombinant human TGF-b3. Alcian blue staining was utilized to examine the cartilage nodules.

\section{Isolation and identification of exosomes}

Exosomes were isolated from the culture medium of DPSCs cultured in the presence of either growth (UN- 
Exo) or odontogenic differentiation media (OD-Exo) for a period of 10 days. Exosomes were isolated as per previously published protocols $[16,17]$. Briefly, 2 days prior to isolation, the cell cultures were washed in serum-free PBS and cultured for $48 \mathrm{~h}$ in serum-free aMEM. When odontogenic media were used, the serumfree a-MEM was supplemented with the odontogenic media cocktail of $100 \mathrm{nmol}$ dexamethasone, $10 \mathrm{mmol} \beta$ glycerophosphate, and $0.2 \mathrm{mmol} \mathrm{L}$-ascorbic acid (Sigma, USA). The exosomes from the culture medium were isolated using the Exo-spin (Cell Guidance, UK) exosome isolation reagent as per the manufacturer's protocol. The exosome protein concentration was quantified with a BCA Protein Assay Kit (Bocai, Shanghai, China). The exosomal markers CD9 and CD63 (Affinity Biosciences, USA) in the UN-Exo and OD-Exo were measured by automated western blot analysis.

\section{Transmission electron microscopy (TEM)}

TEM was used to identify the presence of UN-Exo and OD-Exo. $10 \mu \mathrm{l}$ exosomes suspension was placed on to formvar/carbon-coated nickel TEM grids and incubated for $30 \mathrm{~min}$. The grids were then washed, dried, and imaged using an H-7650 transmission electron microscope (HITACHI, Japan) to identify the morphology of exosomes.

\section{Fluorescent labeling of exosomes}

UN-Exo and OD-Exo were collected from supernatants of DPSC and isolated by ultracentrifugation and sucrose cushion centrifugation. Briefly, cell culture supernatant $(48 \mathrm{~h}$, serum-free medium) was cleared $(2 \times 10 \mathrm{~min}$, $500 \times g ; 1 \times 20 \mathrm{~min}, 2000 \times g ; 1 \times 30 \mathrm{~min}, 10,000 \times g$ ), centrifuged (90 min, 100,000 $\times g$ ), washed (PBS, $90 \mathrm{~min}, 100$, $000 \times g$ ), and further purified by sucrose-gradient centrifugation. $1 \mu \mathrm{l}$ PKH26 (Sigma-Aldrich, St Louis, MO) was added to $250 \mu \mathrm{l}$ diluent $\mathrm{C}$, which was then immediately mixed with the exosomes by pipetting. After 5 min of incubation at room temperature, the staining was stopped by the addition of an equal volume of exosome-free FBS. The exosomes were harvested and washed twice with PBS by centrifugation $(100,000 \mathrm{~g}$ for $1 \mathrm{~h})$ and resuspended in $100 \mu \mathrm{l}$ exosome-free culture medium. The PKH26-labeled exosomes were added to the DPSCs and incubated for $24 \mathrm{~h}$. Then, the cells were washed 3 times with PBS, fixed with $4 \%$ paraformaldehyde for $10 \mathrm{~min}$, and stained with 4, 6-diamidino-2-phenylindole for 5 min. Confocal laser scanning microscopy (Zeiss, Oberkochen, Germany) was used to visualize the endocytosis of UN-Exo and OD-Exo by DPSCs.

Exosome-mediated odontogenic differentiation of DPSCs DPSCs were seeded into 6-well plates at an initial density of $1 \times 10^{5}$ cells/well and incubated for $48 \mathrm{~h}$ with exosomes isolated from DPSCs cultured for 10 days using growth media (UN-Exo, $30 \mu \mathrm{g} / \mathrm{ml}$ ) as well as odontogenic differentiation media (OD-Exo, $30 \mu \mathrm{g} / \mathrm{ml}$ ), the growth media supplemented with $10 \%$ exosome-free FBS was used as control medium. Odontogenic differentiation was measured by automated western blot to explore the protein expression of ALP, RUNX2, odontoblast-specific marker DSP, and DMP-1.

\section{Automated western blot analysis}

The automated western blot was performed using Simple Wes (Protein Simple, USA) following the manufacturer's protocol. Briefly, $1.5 \mu \mathrm{g}$ of protein from the cell lysates or exosomes was added to the standard fluorescent mastermix, then was loaded into corresponding wells of the prefilled Wes assay plate, along with antibody diluent (Protein Simple, USA), anti-DSP (Santa Cruz, USA), anti-DMP1, anti-RUNX2, anti-ALP, antiCD9, anti-CD63, anti-TGF $\beta 1$, anti-TGFR1, anti-Smad2/ 3 , anti-p-Smad2/3, anti-Smad4, anti- $\beta$-Tublin (Affinity, USA), anti-LTBP1 (Affinity, USA), anti-rabbit secondary antibody (Protein Simple, USA), and StreptavidinHRP, followed by luminal peroxide mix. The imaging and analysis were done with compass software (Protein Simple, USA).

\section{microRNA sequencing}

MicroRNAs of 18-30 nucleotides (nt) were obtained from $100 \mu \mathrm{g}$ of total RNA isolated from exosomes using $15 \%$ denaturing polyacrylamide gel electrophoresis (PAGE). After PCR amplification, the products were purified and submitted for sequencing via an Illumina Hi-Seq 2000 platform. Library preparation and microRNA sequencing were performed by RiboBio Ltd. (Guangzhou, China). Differentially expressed microRNAs with 2 fold change in expression $(p<0.05)$ were analyzed. Predicted microRNA target genes were detected using four publicly available bioinformatics tools (TargetScan, miRTarBase, miRDB, and miRWalk databases). Gene Ontology (GO, http://www.geneontology. org) analysis and Kyoto Encyclopedia of Genes and Genomes (KEGG, http://www.genome.jp/kegg/pathway. html) Pathway analysis were performed to detect molecular functions, biological processes, and pathways associated with the predicted microRNA target genes.

$\mathrm{GO}$ analysis, all the targeted genes of miRNAs were input into the Gene Ontology database, and each term number of genes was calculated. Compared with the whole genome background, the hypergeometric test was used to find out the GO terms that were significantly enriched in the targeted genes of miRNAs. The formula is as follows: $p=1-\sum_{i=0}^{m-1} \frac{\left(\frac{M}{i}\right)\left(\frac{N-M}{n-i}\right)}{\left(\frac{N}{n}\right)}$, where $N$ is the number of all genes with GO annotation, $n$ is the number of 
targeted genes of miRNAs in $N, M$ is the number of all genes that are annotated to certain GO terms, $m$ is the number of targeted genes of miRNAs in $M$. The calculated $p$ value goes through Bonferroni correction, taking corrected $p$ value $<0.05$ as a threshold. GO terms fulfilling this condition are defined as significantly enriched GO terms in targeted genes of miRNAs. This analysis is able to recognize the main biological functions that targeted genes of miRNA exercise.

KEGG, the major public pathway-related database, is used to perform pathway enrichment analysis of targeted genes of miRNAs. This analysis identifies significantly enriched metabolic pathways or signal transduction pathways in targeted genes of miRNAs comparing with the whole genome background. The calculating formula is the same as that in the GO analysis. Here, $N$ is the number of all genes that with KEGG annotation, $n$ is the number of targeted genes of miRNAs in $N, M$ is the number of all genes annotated to specific pathways, and $m$ is the number of targeted genes of miRNAs in $M$.

\section{Real-time PCR}

Total RNA was extracted from cells by RNA extraction kit (Qiagen, China). Then, $2 \mu \mathrm{g}$ of total RNA was reversely transcribed into CDNA using a reverse transcription polymerase chain reaction (RT-PCR) system (Promega, USA). The qRT-PCR was performed by SYBR-Green PCR kit (Qiagen, China) according to the manufacturer's instructions on a LightCycler 480 (Roche, USA). The data are representative of three independent experiments, and the relative microRNA expression was determined using the comparative Ct $(\Delta \Delta \mathrm{Ct})$ method. The sequences of the primers are shown in Additional file 1: Table S1.

\section{Determination and inhibition of TGF $\beta 1 /$ smads signaling pathway}

For evaluation of the involvement of TGF $\beta 1 /$ smads signaling pathway, DPSCs were seeded into 6-well plates at an initial density of $1 \times 10^{5}$ cells/well and were incubated for $48 \mathrm{~h}$ with $30 \mu \mathrm{g} / \mathrm{ml} \mathrm{UN-Exo} \mathrm{and} 30 \mu \mathrm{g} / \mathrm{ml}$ OD-Exo, the growth media supplemented with $10 \%$ exosomesfree FBS was used as the control medium. TGF $\beta 1 /$ smads signaling pathway was inhibited by $10 \mu \mathrm{M}$ inhibitor SB525334 (Selleck, USA) for $48 \mathrm{~h}$.

\section{Transfection of miR-27a-5p mimics and inhibitor}

DPSCs were treated with OD-Exo in 6-well culture plates and transfected with the miR-27a-5p mimics and inhibitor using Lipofectamine 2000 (Invitrogen, USA) according to the manufacturer's instructions. DPSCs were harvested after $48 \mathrm{~h}$.

\section{Dual-luciferase reporter assay}

A luciferase reporter gene assay was used to verify whether LTBP1 was the direct target gene of miR-27a-5p. Luciferase reporter constructs encoding the wild-type 3'UTRs of LTBP1 (LTBP1-WT) or mutant 3'-UTRs of AXL (LTBP1-MUT) were synthesized. The 3'-UTR luciferase vector (150 ng) was co-transfected into cells with either miR-27a-5p mimic or miR-27a-5p mimic-control using Lipofectamine 2000 (Invitrogen). After incubation for 48 $\mathrm{h}$, the cells were collected and lysed, and their luciferase activities were detected by the Dual-Luciferase Reporter Assay Kit (Beyotime Biotechnology, Shanghai, China) according to the manufacturer's protocol.

\section{Statistical analysis}

Each experiment was repeated three times. All values were expressed as the mean \pm SD and were evaluated by the independent samples $t$ test using SPSS 17.0 (SPSS Inc., USA). $p<0.05$ was considered statistically significant.

\section{Results}

Characterization of DPSCs

The results showed that DPSCs had the potential of differentiation into osteoblasts, adipocytes, and chondrocytes (Fig. 1a), indicating the multi-lineage differentiation potential of DPSCs. DPSCs expressed high levels of the mesenchymal stem cell marker CD73 (Fig. 1b), CD90 (Fig. 1c), and CD166 (Fig. 1d), but expressed low levels of the hematopoietic cell marker CD45(Fig. 1e).

\section{Endocytosis of UN-Exo and OD-Exo by DPSCs}

To characterize the presence of exosomes in the isolates, the bilayer membrane and "saucerlike" appearance of representative exosomes were examined by TEM, which verified the presence of UN-Exo and OD-Exo ranging from 30 to $150 \mathrm{~nm}$ in diameter (Fig. 2a). Automated western blot analysis revealed that exosomal markers CD9 and CD63 were expressed in the UN-Exo and ODExo (Fig. 2b). To confirm whether UN-Exo and OD-Exo could be taken up by DPSCs, the isolated UN-Exo and OD-Exo were labeled with PKH26, and DPSC cultures were incubated with the labeled exosomes at $37{ }^{\circ} \mathrm{C}$. After 24h, PKH26-labeled UN-Exo and OD-Exo were taken up by DPSCs into the cytoplasm (Fig. 2c).

\section{Endocytosis of OD-Exo by DPSCs triggered odontogenic differentiation}

We evaluated whether the endocytosis of exosomes triggered odontogenic differentiation of DPSCs by affecting the expression of regulatory proteins. When DPSCs were treated with OD-Exo for $48 \mathrm{~h}$, the protein expressions of DSP, DMP-1, ALP, and RUNX2 significantly increased, compared to the control group and UN-Exo treated group $(p<0.05)$. However, DPSCs treated with UN-Exo 


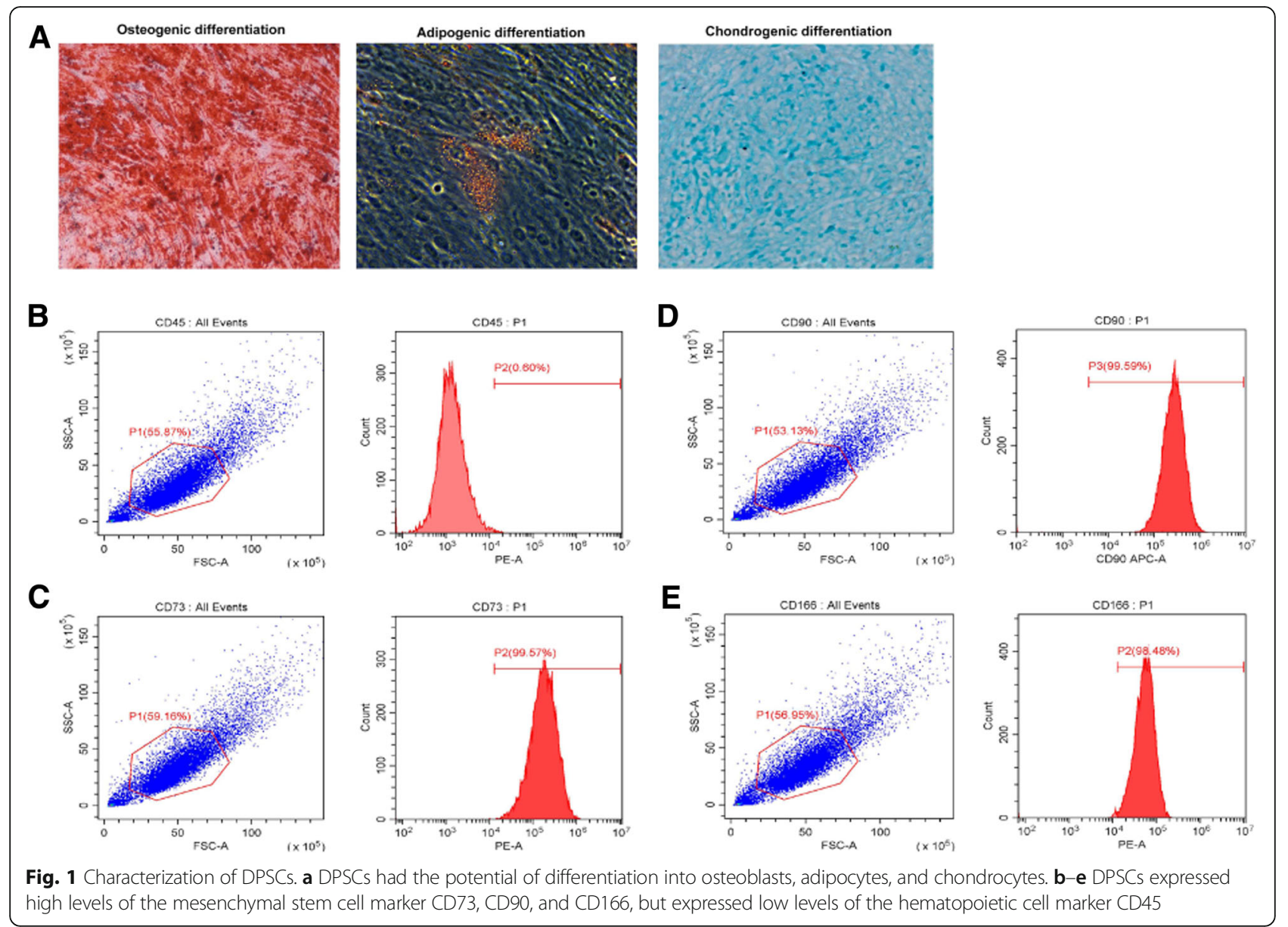

only expressed a higher level of DSP than the control group $(p<0.05)$, but no significant differences in DMP1, ALP, and RUNX2 (Fig. 3). These results showed that OD-Exo isolated under odontogenic conditions are better inducers of DPSCs differentiation.

\section{MicroRNA profiles of UN-Exo and OD-Exo}

As exosomes transfer microRNAs between cells, posttranscriptional gene expression in recipient cells can be regulated by microRNAs contained in exosomes [18]. Thus, we hypothesized that this process may be exploited by DPSCs to promote odontogenic differentiation. To explore this possibility, we analyzed the microRNA profiles of UN-Exo and OD-Exo via Ion Torrent/ MiSeq sequencing. The results showed microRNA levels in OD-Exo significantly changed when compared with that in UN-Exo. There were 28 microRNAs significantly changed in OD-Exo isolated under odontogenic conditions, of which 7 microRNAs increased (miR-5100, miR27a-5p, miR-652-3p, miR-1260a, miR-1260b, let-7f-1-3p, and miR-370-3p) and 21 microRNAs decreased (miR193a-5p, miR-4792, miR-505-3p, miR-629-5p, miR-1403p, miR-185-5p, miR-146b-5p, miR-339-5p, miR-1246,
miR-107, miR-320d, miR-451a, miR-215-5p, miR-1263p, miR-3687, miR-31-5p, miR-210-3p, miR-1-3p, miR10a-5p, miR-10b-5p, and miR-619-5p) (Fig. 4a, Table 1). The qRT-PCR analysis showed that miR-5100 and miR1260a levels in OD-Exo increased, while miR-210-3p and miR-10b-5p decreased, which were consistent with the microRNA sequencing (Fig. 4e).

\section{Pathway and GO analysis of genes targeted by differentially expressed microRNAs}

Four publicly available bioinformatics tools (TargetScan, miRTarBase, miRDB, and miRWalk) were used to analyze genes targeted by differentially expressed microRNAs, for example, miR-27a-5p (Fig. 4b). All genes targeted by differentially expressed microRNAs were shown in Fig. 4c and Additional file 2: Table S2. Pathway analysis showed targeted genes involved in multiple signal transductions, including TGF $\beta$ signaling pathway (Fig. 5a). 16 genes in TGF $\beta$ signaling targeted by 15 differentially expressed microRNAs were shown in Fig. 4d. GO analysis of targeted genes showed that the most significant biological processes consisted of DNA binding, 
A
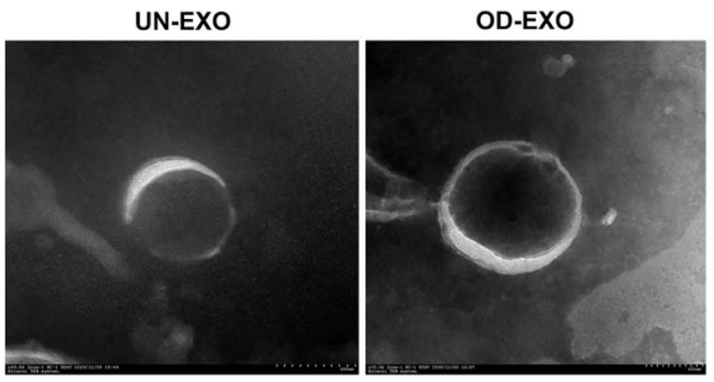

C

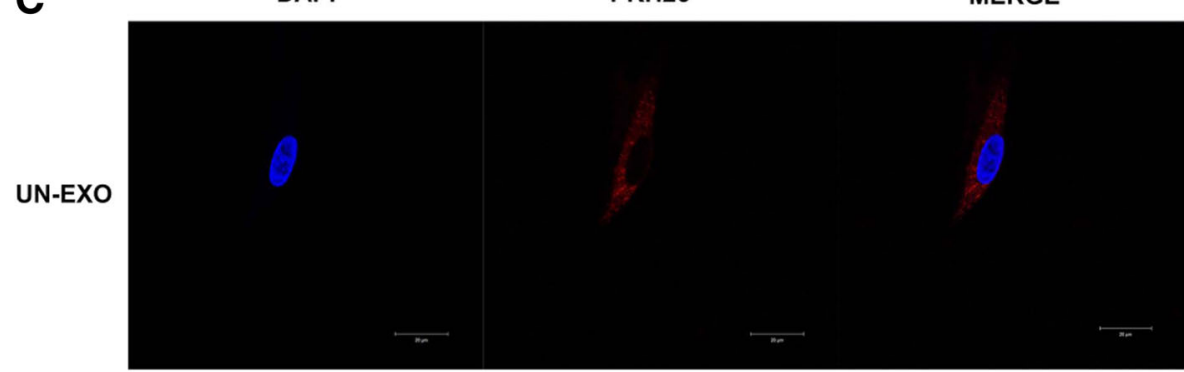

OD-EXO

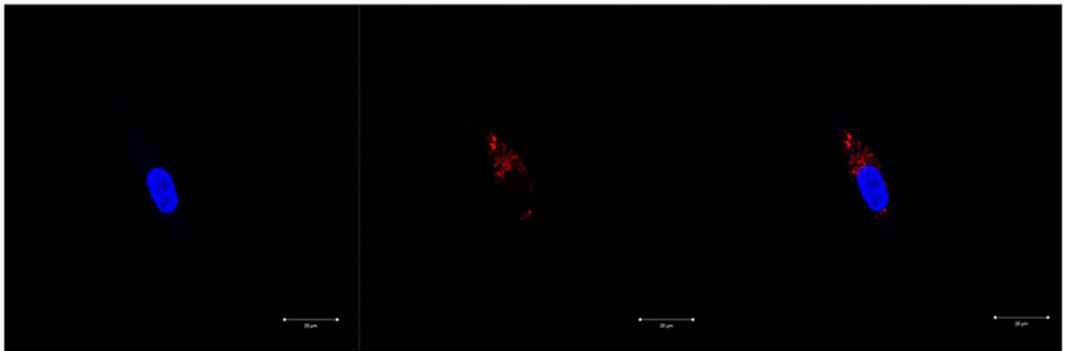

Fig. 2 Endocytosis of UN-EXo and OD-Exo by DPSCS. a The morphology of UN-EXo and OD-Exo was determined by transmission electron microscopy. b Automated western blot analysis revealed that exosomal markers CD9 and CD63 were expressed in the UN-ExO and OD-Exo. $\mathbf{c}$ Endocytosis of exosomes by DPSCs was visualized by fluorescent labeling with PKH26

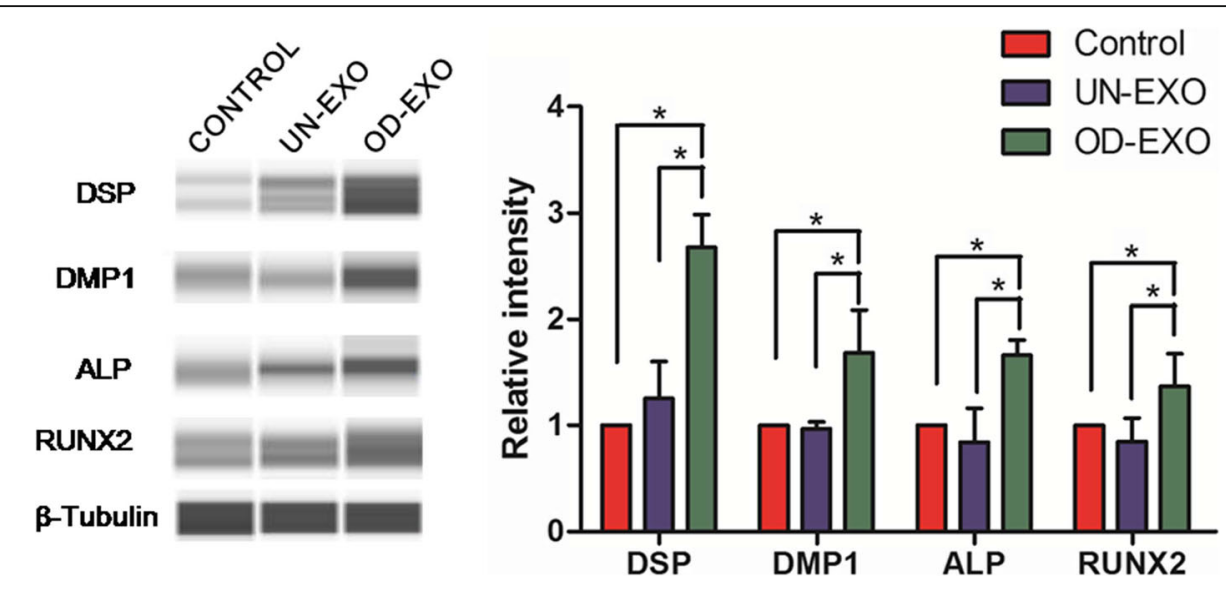

Fig. 3 Endocytosis of OD-Exo by DPSCs triggered odontogenic differentiation. Endocytosis of OD-Exo isolated under odontogenic conditions triggered odontogenic differentiation of DPSCs by upregulating protein expressions of DSP, DMP-1, ALP, and RUNX2, when compared to control group (without exosomes) and UN-Exo group $(p<0.05)$. And DPSCs treated with UN-Exo only expressed higher protein level of DSP than the control group $(p<0.05)$, but no significant differences in DMP-1, ALP, and RUNX2 

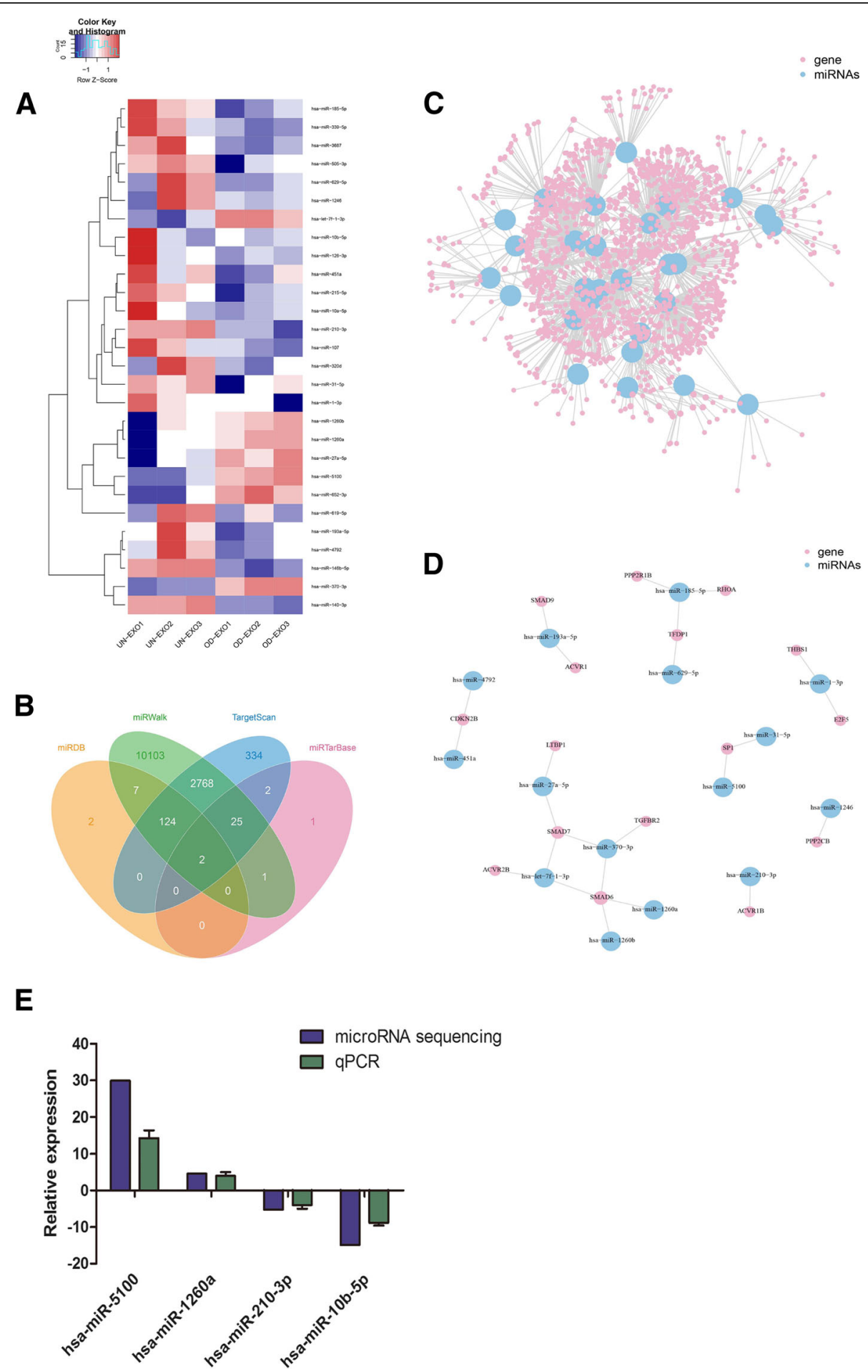

Fig. 4 microRNA profiles of UN-Exo and OD-Exo via microRNA sequencing. a MicroRNA levels in OD-Exo significantly changed when compared with that in UN-EXo, 28 microRNAs significantly changed, of which 7 increased and 21 decreased. b Four bioinformatics tools (TargetScan, miRTarBase, miRDB, and miRWalk) were used to analyze genes targeted by differentially expressed microRNAs, for example, hsa-miR-27a-5p. c All genes targeted by differentially expressed microRNAs were shown in the mRNA-microRNA network. $\mathbf{d}$ mRNA-microRNA network showed 16 genes in TGF $\beta$ signaling were targeted by 15 differentially expressed microRNAs. e Consistent with the microRNA sequencing, hsa-miR-5100 and hsa-miR-1260a were increased; hsa-miR-210-3p and hsa-miR-10b-5p were decreased by qPCR analysis 
Table 1 There were 28 microRNAs significantly changed in ODExo isolated under odontogenic conditions, of which 7 increased and 21 decreased

\begin{tabular}{|c|c|c|c|}
\hline miRNA_ID & Up/down & Fold change & Significance \\
\hline hsa-miR-5100 & Up & 29.91 & ** \\
\hline hsa-miR-27a-5p & Up & 11.42 & $* *$ \\
\hline hsa-miR-652-3p & Up & 10.62 & * \\
\hline hsa-miR-1260a & Up & 4.65 & $* *$ \\
\hline hsa-miR-1260b & Up & 4.00 & $* *$ \\
\hline hsa-let-7f-1-3p & Up & 2.61 & $* *$ \\
\hline hsa-miR-370-3p & Up & 2.54 & ** \\
\hline hsa-miR-193a-5p & Down & 2.09 & * \\
\hline hsa-miR-4792 & Down & 2.13 & * \\
\hline hsa-miR-505-3p & Down & 2.27 & * \\
\hline hsa-miR-629-5p & Down & 2.35 & * \\
\hline hsa-miR-140-3p & Down & 2.72 & $* *$ \\
\hline hsa-miR-185-5p & Down & 2.79 & * \\
\hline hsa-miR-146b-5p & Down & 2.94 & $* *$ \\
\hline hsa-miR-339-5p & Down & 3.08 & * \\
\hline hsa-miR-1246 & Down & 3.56 & * \\
\hline hsa-miR-107 & Down & 3.65 & * \\
\hline hsa-miR-320d & Down & 3.79 & * \\
\hline hsa-miR-451a & Down & 4.20 & * \\
\hline hsa-miR-215-5p & Down & 4.39 & $* *$ \\
\hline hsa-miR-126-3p & Down & 4.44 & * \\
\hline hsa-miR-3687 & Down & 4.64 & $* *$ \\
\hline hsa-miR-31-5p & Down & 4.93 & * \\
\hline hsa-miR-210-3p & Down & 5.23 & $* *$ \\
\hline hsa-miR-1-3p & Down & 9.15 & * \\
\hline hsa-miR-10a-5p & Down & 11.39 & $* *$ \\
\hline hsa-miR-10b-5p & Down & 14.91 & $* *$ \\
\hline hsa-miR-619-5p & Down & 17.70 & $* *$ \\
\hline
\end{tabular}

${ }^{*} p<0.05,{ }^{* *} p<0.01$

catalytic activity, cellular metabolic processes, and regulation of cellular processes (Fig. 5b).

\section{Exosomal microRNAs promoted odontogenic differentiation via TGF $\beta 1 /$ smads signaling pathway by downregulating LTBP1}

To confirm the results of pathway analysis as to whether TGF $\beta$ signaling was activated by exosomal microRNAs encapsulated in OD-Exo, we performed the automated western blot to evaluate the key proteins in TGF $\beta$ signaling. Consistent with pathway analysis, automated western blot found that OD-Exo activated TGF $\beta 1$ pathway by upregulating TGF $\beta 1$, TGFR1, p-Smad2/3, and Smad4 in DPSCs, compared to control group and UN-Exotreated group (Fig. 6a). Accordingly, once we inhibited the TGF $\beta 1$ signaling pathways by SB525334, protein levels of $\mathrm{p}-\mathrm{Smad} 2 / 3$, DSP, and DMP-1 were significantly reduced in DPSCs during odontogenic differentiation induced by OD-Exo for $48 \mathrm{~h}$ (Fig. 6b).

MiR-27a-5p was expressed 11 times higher in OD-Exo isolated under odontogenic conditions (Table 1) and was predicted to be involved in TGF $\beta$ pathway (Fig. 4d). Automated western blot showed that miR-27a-5p mimics promoted odontogenic differentiation of DPSCs by upregulating the protein expressions of DSP, DMP-1, ALP, and RUNX2 (Fig. 7a), and activated TGF $\beta 1 /$ smads signaling pathway by increasing TGF $\beta 1$, TGFR1, p-Smad2/ 3, and Smad4 proteins (Fig. 7b).

As is known, exosomal microRNAs can negatively regulate gene expression by binding to their target mRNAs through base pairing to the 3 '-UTR, there may exist some other inhibitory molecules between the miR$27 \mathrm{a}-5 \mathrm{p}$ and TGF $\beta 1$ signaling. Latent TGF- $\beta$-binding protein 1 (LTBP1), one of the inhibitory molecules of TGF $\beta 1$ signaling, which form latent complexes with TGF $\beta$ by covalently binding the TGF $\beta$ propeptide (LAP) via disulfide bonds, plays a role in maintaining TGF $\beta$ latency by anchoring TGF- $\beta$ to the extracellular matrix [19]. It was found that the mutation of LTBP-1 in mice resulted in excess active TGF- $\beta$, which caused increased signaling through its receptor and accumulation of nuclear p-Smad2/3 [20]. Using four publicly available bioinformatics tools, LTBP1 was found to be targeted by miR-27a-5p (Fig. 4b, d). We used western blot and double luciferase assay to test whether LTBP1 could be directly downregulated by miR-27a-5p. Western blot showed that LTBP1 was downregulated by miR-27a-5p mimics (Fig. 7c). Figure 7d showed the predicted miRNA binding sites in the 3 '-UTR of LTBP1. The luciferase reporter assay was used to determine whether miR-27a-5p could target the 3 '-UTR of LTBP1 directly. The 3 '-UTR fragment (LTBP1-WT) of LTBP1 containing a miR-27a$5 \mathrm{p}$ binding site and mutant fragments (LTBP1-MUT) was cloned into luciferase reporter vectors. miR-27a-5p was found to significantly reduce LTBP1-WT luciferase activity while it had no effect on the luciferase activity of LTBP1-MUT group (Fig. 7e). Hence, the exosomal microRNAs promoted odontogenic differentiation via TGF $\beta 1 /$ smads signaling pathway by downregulating the inhibitory molecule LTBP1 (Fig. 8).

\section{Discussion}

Regenerative endodontic treatment which has been defined as biologically based procedures designed to replace damaged structures, including dentin and root structures, as well as cells of the pulp-dentin complex, has great potential in treating endodontic disease [21]. Stem cells can be implanted into the root canal via a suitable medium, induced to proliferate, migrate, and 


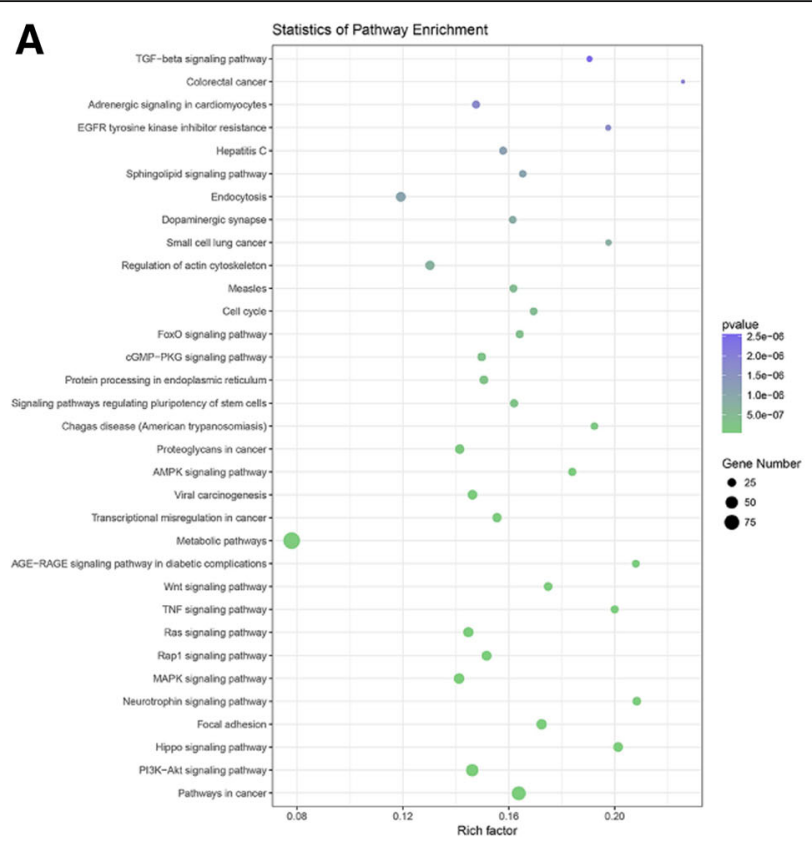

B

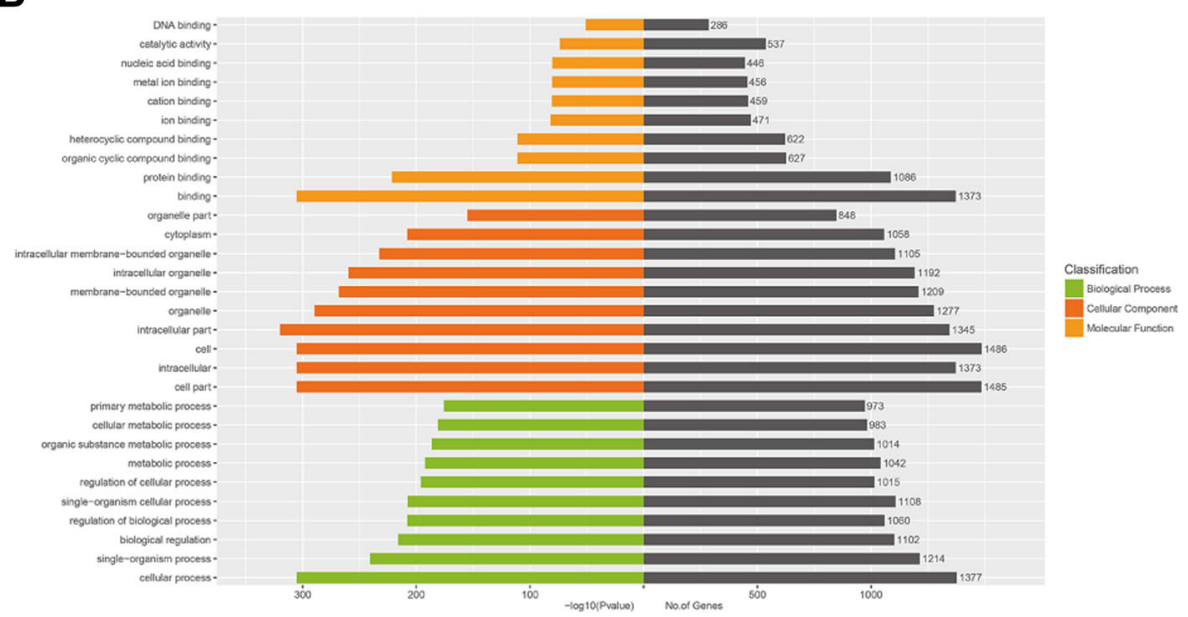

Fig. 5 Pathway and GO analysis of genes targeted by differentially expressed microRNAs. a Pathway analysis showed that targeted genes involved in multiple signal transductions, including TGF $\beta$ signaling pathway. $\mathbf{b}$ GO analysis of targeted genes showed that the most significant biological processes consisted of DNA binding, catalytic activity, cellular metabolic process, and regulation of the cellular process

differentiate into different cell types by biomaterials and growth factors to regenerate damaged tissue [22]. DPSCs is a suitable source for cells in regenerative endodontic treatment, and its differentiation into odontogenic lineage induced by biomaterials and growth factors is essential in dental pulp regeneration [1]. As reported, exosomes can be used as biomimetic tools to induce odontoblast-specific differentiation of DPSCs in regenerative endodontic treatment [14]. The present study determined that endocytosis of exosomes triggered odontogenic differentiation of DPSCs by evaluating the expression of ALP, RUNX2, odontoblastspecific marker DMP-1, and DSP proteins. Moreover, this study showed that exosomes isolated from DPSCs undergoing odontogenic differentiation (OD-Exo) were better inducers of DPSC differentiation than exosomes undergrowth medium (UN-Exo). Consistent with previous studies, cell type-specific exosomes can induce lineage-specific differentiation of stem cells. For example, DPSC-derived exosomes can direct the differentiation of the bone marrow MSCs towards an odontogenic lineage [14]. Similarly, osteoblast-derived exosomes containing instructive factors promoted the osteogenic differentiation of MSCs, while the adipocyte-derived exosomes triggered adipogenic differentiation of MSCs [7]. 


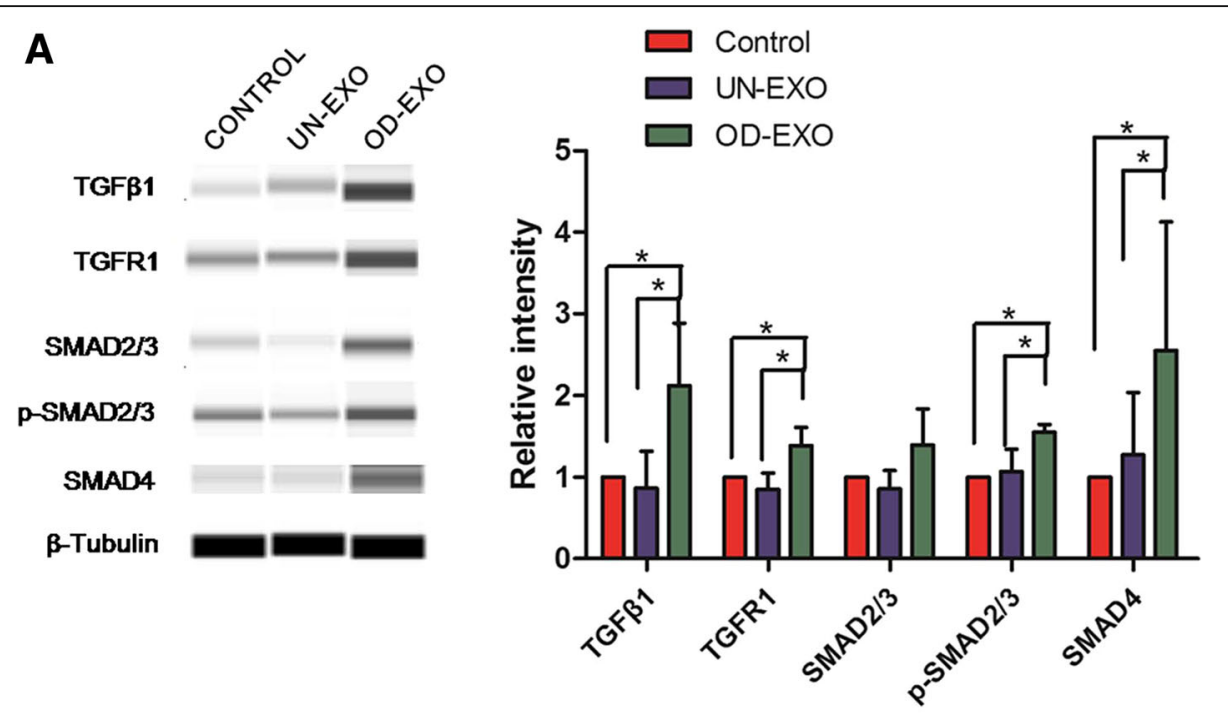

$\mathbf{B}$

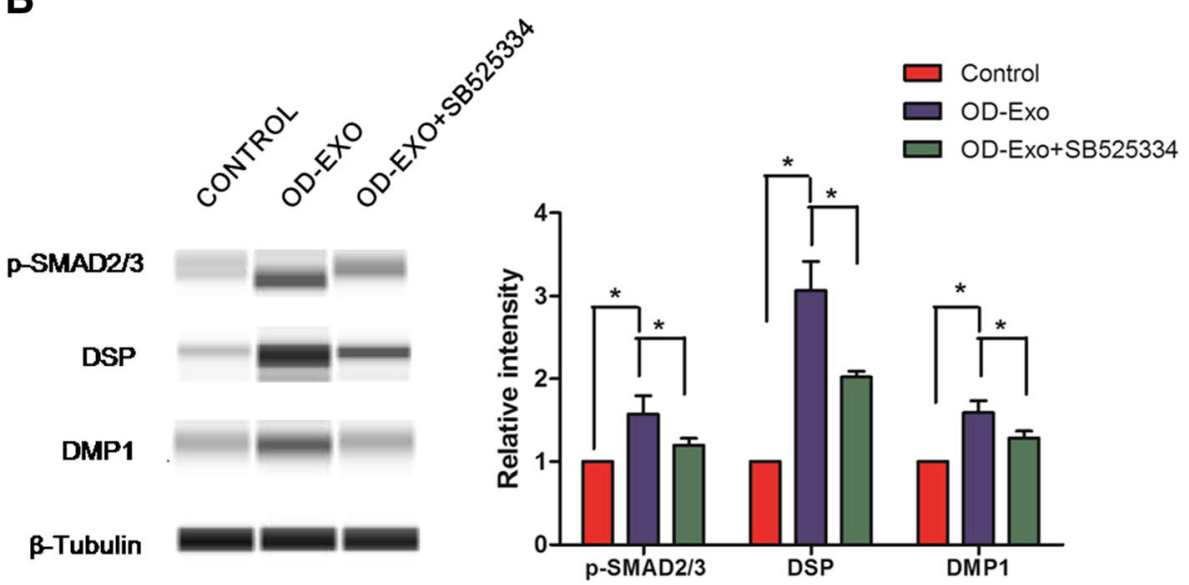

Fig. 6 OD-Exo promoted odontogenic differentiation via TGF $\beta 1 /$ smads signaling pathway. a Consistent with pathway analysis, automated western blot analysis found significantly upregulated protein expressions of TGF $\beta 1$, TGFR1, p-Smad2/3, and Smad4 in DPSCs treated with OD-Exo, when compared to the control group and UN-EXo-treated group. $\mathbf{b}$ In accordance, once we inhibited the TGF 31 signaling pathways by SB525334, protein levels of p-Smad2/3, DSP, and DMP-1 were significantly reduced in DPSCs treated with OD-Exo

Exosomes, by carrying cell-type specific biological molecules such as protein, mRNA, and microRNA, serve as a mode of intercellular communications during tissue formation and repair [7]. Exosomal microRNAs can negatively regulate gene expression by binding to their target mRNAs through base pairing to the $3^{\prime}$-UTR, causing translational repression of the mRNA in recipient cells [23]. Exosomal microRNAs play important roles in stem cell differentiation [24]; however, the function of microRNAs encapsulated in exosomes derived from DPSCs is still unrevealed. In the present study, we performed microRNA sequencing to clarify microRNA expression profiles of exosomes derived from DPSCs. The results showed microRNA levels in OD-Exo were significantly changed when compared with that in UN-Exo.
There were 28 microRNAs significantly changed in ODExo isolated under odontogenic conditions, of which 7 microRNAs increased (miR-5100, miR-27a-5p, miR-6523p, miR-1260a, miR-1260b, let-7f-1-3p, and miR-370-3p) and 21 microRNAs decreased (miR-193a-5p, miR-4792, miR-505-3p, miR-629-5p, miR-140-3p, miR-185-5p, miR-146b-5p, miR-339-5p, miR-1246, miR-107, miR320d, miR-451a, miR-215-5p, miR-126-3p, miR-3687, miR-31-5p, miR-210-3p, miR-1-3p, miR-10a-5p, miR10b-5p, and miR-619-5p). Among these, 2 upregulated microRNAs (miR-5100 [25], miR-652-3p [26]), and 5 downregulated microRNAs (miR-185-5p [27], miR-107 [28], miR-215-5p [29], miR-31-5p [30], and miR-10b-5p [31]) have been found to play important roles in stem cell differentiation. miR-5100 has been verified to be 
A
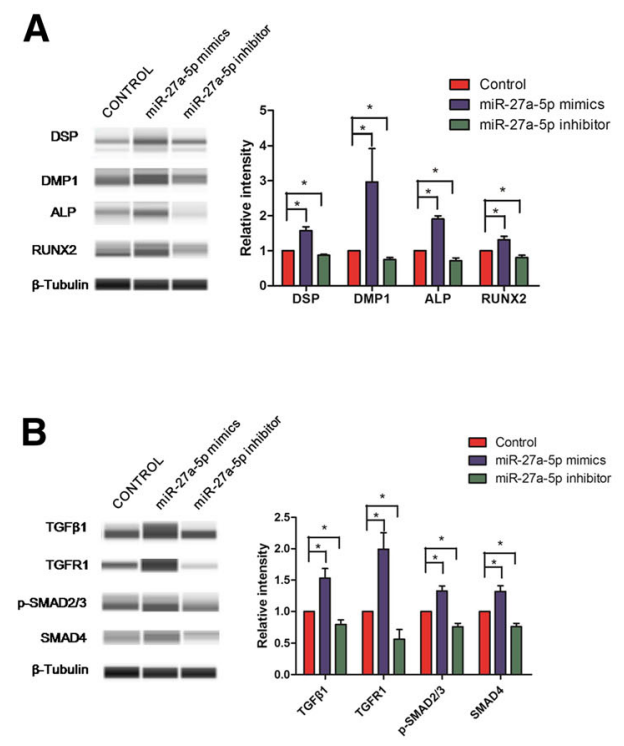

C

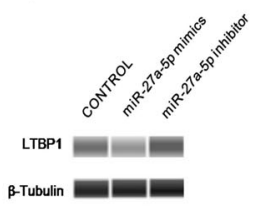

D

MIR-27a-5p 3-ACGAGUGUUCGUCGAUUCGGGAS

LTBP13'UTR 5'-AGAAUCUACAUAACGUAMGOCCA-3'

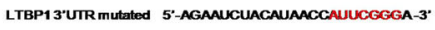

E

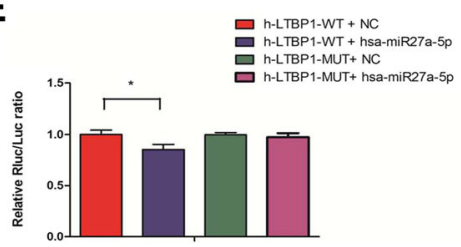

Fig. 7 Exosomal miR-27a-5p regulated odontogenic differentiation via TGF $31 /$ smads signaling pathway by downregulating LTBP1. a miR-27a-5p mimics promoted odontogenic differentiation of DPSCs by upregulating the protein expressions of DSP, DMP-1, ALP, and RUNX2. $\mathbf{b}$ miR-27a-5p mimics activated TGF $\beta 1 /$ smads signaling pathway by increasing TGF $\beta 1$, TGFR1, p-Smad2/3, and Smad4 proteins. $\mathbf{c}$ The western blot showed LTBP1 was downregulated bymiR-27a-5p mimics. $\mathbf{d}$ The predicted miRNA binding sites in the 3'-UTR of LTBP1. e Luciferase reporter assay found that miR27a-5p could significantly reduce LTBP1-WT luciferase activity and that miR-27a-5p had no effect on the luciferase activity of LTBP1-MUT group

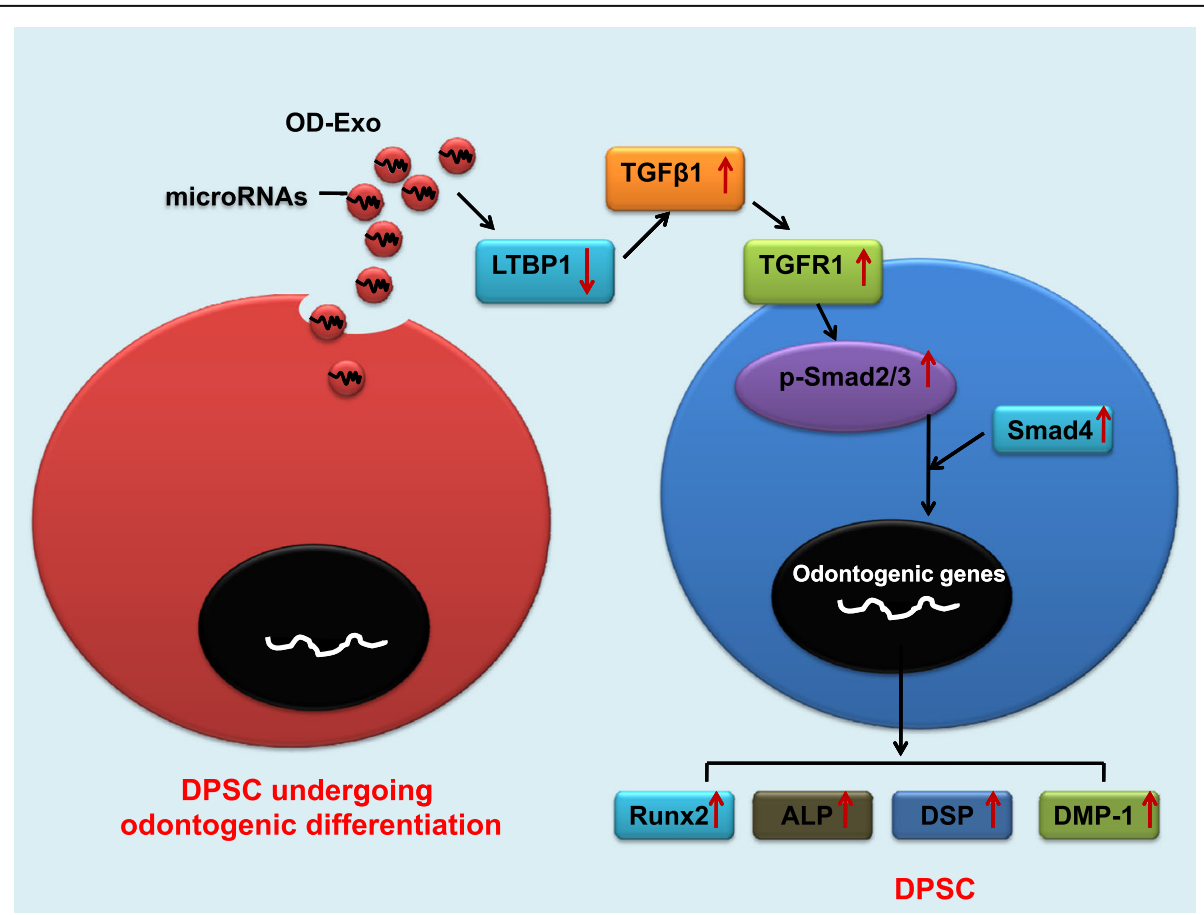

Fig. 8 Summary of the function of OD-Exo in the odontogenic differentiation of DPSCS through TGF $\beta 1 /$ smads signaling pathway via the transfer of mircoRNAs 
upregulated during osteoblast differentiation and promoted osteogenic differentiation of MSCs [25]. miR-652$3 p$ has been found significantly upregulated during neuronal differentiation and might play a pivotal role in the promotion of neuronal differentiation [26]. On the other hand, suppression of miR-185-5p enhanced ameloblast differentiation of LS8 cells and osteogenesis of MC3T3E1 cells by regulating Dlx2 expression [27]. miR-107 was found to inhibit myoblast differentiation [28] and adipocyte differentiation [32]. miR-215-5p inhibited adipocyte differentiation of 3 T3-L1 cells through posttranscriptional regulation of fibronectin type III domain containing 3B (FNDC3B) and catenin-beta interacting protein 1 (CTNNBIP1) during early adipogenesis [29]. miR-31-5p inhibition enhanced the adipogenic differentiation in hADSCs, since miR-31-5p directly bound to the 3'-UTR of C/EBP- $\alpha$ to inhibit its expression [30]. Downregulation of miR-10b-5p promoted the differentiation of 3 T3-L1 cells and adipogenesis by upregulating the Apol6 expression [31]. In the present study, we transfected DPSCs with miR-27a-5p which was expressed 11 times higher in OD-Exo, and the results show miR-27a-5p mimics promoted odontogenic differentiation of DPSCs by upregulating the protein expressions of DSP, DMP-1, ALP, and RUNX2. Consequently, we can draw the conclusion that exosomes isolated from DPSCs undergoing odontogenic differentiation (ODExo) promoted the odontogenic differentiation via the transfer of mircoRNAs.

In our study, genes targeted by differentially expressed microRNAs were predicted by four publicly available bioinformatics tools (TargetScan, miRTarBase, miRDB, and miRWalk). Pathway analysis showed genes targeted by differentially expressed microRNAs were involved in multiple signal transductions, including TGF $\beta$ signaling pathway. TGF $\beta$ signaling has been shown to play important roles in odontogenic differentiation and tooth development [33, 34]. TGF $\beta$ signaling pathway was activated by exosomes via upregulating $\mathrm{p}$-Smad2 and promoting nuclear localization of Smad4 [35]. Exosomes regulated the TGF $\beta$ pathway by increasing expression of Smad-3 in liver cells [36]. It has been revealed that exosomes regulated the TGF $\beta$ pathway by transferring exosomal microRNAs. Exosomal miR-302b influenced the TGF $\beta$ pathway by suppressing the expression of TGF $\beta 22$ [37]. Exosomal miR-let7c attenuated kidney injury by significantly downregulated TGF $\beta 1$ and TGFR1 in the kidneys [38]. Exosomal miR-132 promoted lymphangiogenic response by directly targeting Smad-7 and regulating TGF- $\beta /$ Smad signaling [39]. In the present study, there were 16 genes targeted by 15 differentially expressed microRNAs were involved in TGF $\beta$ signaling. To confirm whether TGF $\beta$ signaling was activated by exosomal microRNAs, we evaluated the key proteins in TGF $\beta$ signaling. Consistent with pathway analysis, the protein expressions of TGF $\beta 1$, TGFR1, pSmad2/3, and Smad4 were significantly increased when DPSCs were treated with OD-Exo for $48 \mathrm{~h}$. In accordance, we inhibited the TGF $\beta 1$ signaling pathways by SB525334 and found protein levels of pSmad2/3, DSP, and DMP-1 were significantly reduced in DPSCs during odontogenic differentiation induced by OD-Exo. We transfected DPSCs with miR-27a-5p mimics which was predicted to be involved in TGF $\beta$ pathway, and the results show miR-27a-5p mimics activated TGF $\beta 1 /$ smads signaling pathway by increasing TGF $\beta 1$, TGFR1, $\mathrm{p}-\mathrm{Smad} 2 / 3$, and Smad4 proteins. Thus, the exosomal microRNAs promoted odontogenic differentiation via TGF $\beta 1 /$ smads signaling pathway.

Since exosomal microRNAs negatively regulate gene expression by binding to the 3'-UTRs of their target mRNAs, there may exist some inhibitory molecules between the miR-27a-5p and TGF $\beta 1$ signaling. As reported, latent TGF- $\beta$-binding protein 1 (LTBP1) was one of the inhibitory molecules of TGF $\beta 1$ signaling. The LTBP1 was first identified as forming latent complexes with TGF $\beta$ by covalently binding the TGF $\beta$ propeptide (LAP) via disulfide bonds and was thought to primarily play a role in maintaining TGF $\beta$ latency by anchoring TGF- $\beta$ to the extracellular matrix [19]. Mutation of LTBP-1 in mice resulted in activating TGF- $\beta$ signaling through its receptor and accumulation of nuclear $\mathrm{p}-\mathrm{Smad} 2 / 3$ [20]. We performed western blot and double luciferase assay to test whether LTBP1 could be directly downregulated by miR-27a-5p. Interestingly, the western blot showed LTBP1 protein levels were reduced by miR27a-5p mimics. Moreover, we found that miR-27a-5p could significantly reduce WT-LTBP1 luciferase activity and that miR-27a-5p had no effect on the luciferase activity of MUT-LTBP1 group. Luciferase reporter gene assay verified LTBP1 was the direct target gene of miR-27a-5p. Taken together, we draw a conclusion that the exosomal microRNAs promoted odontogenic differentiation via TGF $\beta 1 /$ smads signaling pathway by downregulating the inhibitory molecule LTBP1.

\section{Conclusions}

This study identified the microRNA expression profiles of exosomes derived from DPSCs. Our results showed that OD-Exo isolated under odontogenic conditions were better inducers of DPSC differentiation. Exosomal microRNAs promoted odontogenic differentiation via TGF $\beta 1 /$ smads signaling pathway by downregulating the inhibitory molecule LTBP1. 


\section{Additional files}

Additional file 1: Table S1. Primer pairs used in the $q R T-P C R$. (DOCX $15 \mathrm{~kb}$ )

Additional file 2: Table S2. All genes targeted by differentially expressed microRNAs were analyze by four publicly available bioinformatics tools (TargetScan, miRTarBase, miRDB, and miRWalk). (XLSX 41 kb)

\section{Abbreviations \\ ALP: Alkaline phosphatase; DMP-1: Dentin matrix protein 1; DPSCs: Dental pulp stem cells; DSP: Dentin sialophosphoprotein; GO: Gene Ontology; LTBP1: Latent TGF- $\beta$-binding protein 1; MSCs: Mesenchymal stem cells; TGFR1: Transforming growth factor beta receptor 1; TGF $\beta 1$ : Transforming growth factor beta 1}

\section{Acknowledgements}

Not applicable.

\section{Authors' contributions}

$\mathrm{JZ}$ and $\mathrm{XH}$ conceived this study. JZ, YZ, and YK performed the experiments, collected data, and performed data analysis. YC and JF helped perform the experiments. JZ and XH wrote the manuscript. All authors read and approved the final manuscript.

\section{Funding}

This work was supported by the National Natural Science Foundation of China (Nos. 11772361 and 81700950), Youth Teacher Training Project of Sun Yat-sen University (No: 17ykpy74).

\section{Availability of data and materials}

The authors confirm that all data generated or analyzed during this study are available.

\section{Ethics approval and consent to participate}

All experiments were approved by the Ethics Committee of Sun Yat-sen University for the use of rat tissue.

\section{Consent for publication}

Not applicable.

\section{Competing interests}

The authors declare that they have no competing interests.

\section{Author details}

'Department of Operative Dentistry and Endodontics, Guanghua School of Stomatology, Affiliated Stomatological Hospital, Sun Yat-sen University, Guangzhou 510055, Guangdong, China. ${ }^{2}$ Guangdong Provincial Key Laboratory of Stomatology, Sun Yat-sen University, Guangzhou, Guangdong, China. ${ }^{3}$ Key Laboratory of Oral Medicine, Guangzhou Institute of Oral Disease, Stomatology Hospital of Guangzhou Medical University, Guangzhou, Guangdong, China. ${ }^{4}$ Department of Endodontics, Stomatology Hospital of Guangzhou Medical University, Guangzhou, Guangdong, China.

Received: 12 February 2019 Revised: 14 May 2019 Accepted: 23 May 2019 Published online: 13 June 2019

\section{References}

1. Xuan K, Li B, Guo H, Sun W, Kou X, He X, Zhang Y, Sun J, Liu A, Liao L, et al. Deciduous autologous tooth stem cells regenerate dental pulp after implantation into injured teeth. Sci Transl Med. 2018;10(455). https://doi.org/ 10.1126/scitransImed.aaf3227

2. Ravindran S, Huang CC, George A. Extracellular matrix of dental pulp stem cells: applications in pulp tissue engineering using somatic MSCs. Front Physiol. 2014:4:395.

3. Yang JW, Zhang YF, Sun ZY, Song GT, Chen Z. Dental pulp tissue engineering with bFGF-incorporated silk fibroin scaffolds. J Biomater Appl. 2015;30(2):221-9.

4. Sun G, Li G, Li D, Huang W, Zhang R, Zhang H, Duan Y, Wang B. hucMSC derived exosomes promote functional recovery in spinal cord injury mice via attenuating inflammation. Mater Sci Eng C Mater Biol Appl. 2018;89:194-204.
5. Li Y, Yang YY, Ren JL, Xu F, Chen FM, Li A. Exosomes secreted by stem cells from human exfoliated deciduous teeth contribute to functional recovery after traumatic brain injury by shifting microglia M1/M2 polarization in rats. Stem Cell Res Ther. 2017;8(1):198.

6. Alcayaga-Miranda F, Varas-Godoy M, Khoury M. Harnessing the angiogenic potential of stem cell-derived exosomes for vascular regeneration. Stem Cells Int. 2016;2016:3409169.

7. Narayanan K, Kumar S, Padmanabhan P, Gulyas B, Wan ACA, Rajendran VM. Lineage-specific exosomes could override extracellular matrix mediated human mesenchymal stem cell differentiation. Biomaterials. 2018;182:312-22.

8. Zhang J, Liu X, Li H, Chen C, Hu B, Niu X, Li Q, Zhao B, Xie Z, Wang Y. Exosomes/tricalcium phosphate combination scaffolds can enhance bone regeneration by activating the PI3K/Akt signaling pathway. Stem Cell Res Ther. 2016;7(1):136.

9. Thery C, Zitvogel L, Amigorena S. Exosomes: composition, biogenesis and function. Nat Rev Immunol. 2002;2(8):569-79.

10. Li YY, Tao YW, Gao S, Li P, Zheng JM, Zhang SE, Liang J, Zhang Y. Cancerassociated fibroblasts contribute to oral cancer cells proliferation and metastasis via exosome-mediated paracrine miR-34a-5p. EBioMedicine. 2018;36:209-20.

11. He L, Hannon GJ. MicroRNAs: small RNAs with a big role in gene regulation. Nat Rev Genet. 2004;5(7):522-31.

12. Sun H, Hu S, Zhang Z, Lun J, Liao W, Zhang Z. Expression of exosomal microRNAs during chondrogenic differentiation of human bone mesenchymal stem cells. J Cell Biochem. 2019;120(1):171-81.

13. Choi SY, Han EC, Hong SH, Kwon TG, Lee Y, Lee HJ. Regulating osteogenic differentiation by suppression of exosomal microRNAs. Tissue Eng A. 2018. https://doi.org/10.1089/ten.TEA.2018.0257.

14. Huang CC, Narayanan R, Alapati S, Ravindran S. Exosomes as biomimetic tools for stem cell differentiation: applications in dental pulp tissue regeneration. Biomaterials. 2016;111:103-15.

15. Gronthos S, Mankani M, Brahim J, Robey PG, Shi S. Postnatal human dental pulp stem cells (DPSCs) in vitro and in vivo. Proc Natl Acad Sci U S A. 2000; 97(25):13625-30.

16. Narayanan R, Huang CC, Ravindran S. Hijacking the cellular mail: exosome mediated differentiation of mesenchymal stem cells. Stem Cells Int. 2016; 2016:3808674.

17. Ti D, Hao H, Tong C, Liu J, Dong L, Zheng J, Zhao Y, Liu H, Fu X, Han W. LPS-preconditioned mesenchymal stromal cells modify macrophage polarization for resolution of chronic inflammation via exosome-shuttled let7b. J Transl Med. 2015;13:308.

18. Phinney DG, Di Giuseppe M, Njah J, Sala E, Shiva S, St Croix CM, Stolz DB, Watkins SC, Di YP, Leikauf GD, et al. Mesenchymal stem cells use extracellular vesicles to outsource mitophagy and shuttle microRNAs. Nat Commun. 2015;6:8472

19. Todorovic V, Rifkin DB. LTBPs, more than just an escort service. J Cell Biochem. 2012;113(2):410-8.

20. Mazzieri R, Jurukovski V, Obata H, Sung J, Platt A, Annes E, KaramanJurukovska N, Gleizes PE, Rifkin DB. Expression of truncated latent TGF-betabinding protein modulates TGF-beta signaling. J Cell Sci. 2005;118(Pt 10): 2177-87.

21. Metlerska J, Fagogeni I, Nowicka A. Efficacy of autologous platelet concentrates in regenerative endodontic treatment: a systematic review of human studies. J Endod. 2019;45(1):20-30 e21.

22. Tong HJ, Rajan S, Bhujel N, Kang J, Duggal M, Nazzal H. Regenerative endodontic therapy in the management of nonvital immature permanent teeth: a systematic review-outcome evaluation and meta-analysis. J Endod. 2017:43(9):1453-64

23. Bhome R, Del Vecchio F, Lee GH, Bullock MD, Primrose JN, Sayan AE, Mirnezami AH. Exosomal microRNAs (exomiRs): small molecules with a big role in cancer. Cancer Lett. 2018:420:228-35.

24. Fang $S$, Xu C, Zhang Y, Xue C, Yang C, Bi H, Qian X, Wu M, Ji K, Zhao Y, et al. Umbilical cord-derived mesenchymal stem cell-derived exosomal microRNAs suppress myofibroblast differentiation by inhibiting the transforming growth factor-beta/SMAD2 pathway during wound healing. Stem Cells Transl Med. 2016;5(10):1425-39.

25. Wang H, Cui Y, Luan J, Zhou X, Li C, Li H, Shi L, Han J. MiR-5100 promotes osteogenic differentiation by targeting Tob2. J Bone Miner Metab. 2017 35(6):608-15.

26. He H, Li W, Peng M, Qin J, Shi J, Li H, Tian M, Zhang X, Lv G, Jin G. MicroRNA expression profiles of neural stem cells following valproate inducement. J Cell Biochem. 2018;119(7):6204-15. 
27. Chang H, Wang Y, Liu H, Nan X, Wong S, Peng S, Gu Y, Zhao H, Feng H. Mutant RunX2 regulates amelogenesis and osteogenesis through a miR185-5p-Dlx2 axis. Cell Death Dis. 2017;8(12):3221.

28. Li H, Wei X, Yang J, Dong D, Hao D, Huang Y, Lan X, Plath M, Lei C, Ma Y, et al. circFGFR4 promotes differentiation of myoblasts via binding miR-107 to relieve its inhibition of Wnt3a. Molecular therapy Nucleic acids. 2018;11: 272-83

29. Peng Y, Li H, Li X, Yu S, Xiang H, Peng J, Jiang S. MicroRNA-215 impairs adipocyte differentiation and co-represses FNDC3B and CTNNBIP1. Int J Biochem Cell Biol. 2016;79:104-12.

30. Liu Y, Wang Y, He X, Zhang S, Wang K, Wu H, Chen L. LncRNA TINCR/miR31-5p/C/EBP-alpha feedback loop modulates the adipogenic differentiation process in human adipose tissue-derived mesenchymal stem cells. Stem Cell Res. 2018;32:35-42.

31. Ge G, Yang D, Tan Y, Chen Y, Jiang D, Jiang A, Li Q, Liu Y, Zhong Z, Li X, et al. miR-10b-5p regulates $\mathrm{C} 2 \mathrm{C} 12$ myoblasts proliferation and differentiation. Biosci Biotechnol Biochem. 2019;83(2):291-299.

32. Ahonen MA, Haridas PAN, Mysore R, Wabitsch M, Fischer-Posovszky P, Olkkonen VM. miR-107 inhibits CDK6 expression, differentiation, and lipid storage in human adipocytes. Mol Cell Endocrinol. 2019;479:110-6.

33. He XY, Sun K, Xu RS, Tan JL, Pi CX, Wan M, Peng YR, Ye L, Zheng LW, Zhou $X D$. Spatial signalling mediated by the transforming growth factor-beta signalling pathway during tooth formation. Int J Oral Sci. 2016;8(4):199-204.

34. He W, Zhang J, Niu Z, Yu Q, Wang Z, Zhang R, Su L, Fu L, Smith AJ, Cooper PR. Regulatory interplay between NFIC and TGF-beta1 in apical papilladerived stem cells. J Dent Res. 2014;93(5):496-501.

35. Wang $\mathrm{S}, \mathrm{Li}$ X, Xu M, Wang J, Zhao RC. Reduced adipogenesis after lung tumor exosomes priming in human mesenchymal stem cells via TGFbeta signaling pathway. Mol Cell Biochem. 2017:435(1-2):59-66.

36. Koeck ES, lordanskaia T, Sevilla S, Ferrante SC, Hubal MJ, Freishtat RJ, Nadler EP. Adipocyte exosomes induce transforming growth factor beta pathway dysregulation in hepatocytes: a novel paradigm for obesity-related liver disease. J Surg Res. 2014;192(2):268-75.

37. Li J, Yu J, Zhang H, Wang B, Guo H, Bai J, Wang J, Dong Y, Zhao Y, Wang Y. Exosomes-derived MiR-302b suppresses lung cancer cell proliferation and migration via TGFbetaRII inhibition. Cel Physiol Biochem. 2016;38(5):1715-26.

38. Wang B, Yao K, Huuskes BM, Shen HH, Zhuang J, Godson C, Brennan EP, Wilkinson-Berka JL, Wise AF, Ricardo SD. Mesenchymal stem cells deliver exogenous microRNA-let7c via exosomes to attenuate renal fibrosis. Mol Ther. 2016;24(7):1290-301.

39. Wang X, Wang H, Cao J, Ye C. Exosomes from adipose-derived stem cells promotes VEGF-C-dependent lymphangiogenesis by regulating miRNA-132/ TGF-beta pathway. Cell Physiol Biochem. 2018;49(1):160-71.

\section{Publisher's Note}

Springer Nature remains neutral with regard to jurisdictional claims in published maps and institutional affiliations.

Ready to submit your research? Choose BMC and benefit from:

- fast, convenient online submission

- thorough peer review by experienced researchers in your field

- rapid publication on acceptance

- support for research data, including large and complex data types

- gold Open Access which fosters wider collaboration and increased citations

- maximum visibility for your research: over $100 \mathrm{M}$ website views per year

At $\mathrm{BMC}$, research is always in progress.

Learn more biomedcentral.com/submissions 